\title{
The prevalence of IgG4-related hypophysitis in 170 consecutive patients with hypopituitarism and/or central diabetes insipidus and review of the literature
}

\author{
Hironori Bando, Genzo Iguchi, Hidenori Fukuoka, Masaaki Taniguchi ${ }^{1}$, \\ Masaaki Yamamoto, Ryusaku Matsumoto, Kentaro Suda, Hitoshi Nishizawa, \\ Michiko Takahashi, Eiji Kohmura' ${ }^{1}$ and Yutaka Takahashi
}

Division of Diabetes and Endocrinology, Department of Internal Medicine and ${ }^{1}$ Department of Neurosurgery, Kobe University Graduate School of Medicine, 7-5-1 Kusunoki-cho, Chuo-ku, Kobe, Hyogo 650-0017, Japan

\author{
Correspondence \\ should be addressed \\ to $Y$ Takahashi \\ Email \\ takahash@med.kobe-u.ac.jp
}

\section{Abstract}

Objective: The prevalence and clinical characteristics of IgG4-related hypophysitis remain unclear due to the limited number of case reports. Therefore, in this study, we screened consecutive outpatients with hypopituitarism and/or diabetes insipidus (DI) to estimate its prevalence.

Methods: A total of 170 consecutive outpatients with hypopituitarism and/or central DI were screened at Kobe University Hospital for detecting IgG4-related hypophysitis by pituitary magnetic resonance imaging, measuring serum IgG4 concentrations, assessing the involvement of other organs, and carrying out an immunohistochemical analysis to detect IgG4-positive cell infiltration.

Results: Among the screened cases, 116 cases were excluded due to diagnosis of other causes such as tumors and congenital abnormalities. Additionally, 22 cases with isolated ACTH deficiency were analyzed and were found not to meet the criteria of IgG4-related hypophysitis. The remaining 32 cases were screened and seven were diagnosed with IgG4-related hypophysitis, of which three cases were diagnosed by analyzing pituitary specimens. IgG4-related hypophysitis was detected in $30 \%$ (seven of 23 patients) of hypophysitis cases and $4 \%$ of all hypopituitarism/DI cases. The mean age at the onset of IgG4-related hypophysitis was $61.8 \pm 8.8$ years, and the serum IgG4 concentration was $191.1 \pm 78.3 \mathrm{mg} / \mathrm{dl}$ (normal values $5-105 \mathrm{mg} / \mathrm{dl}$ and values in IgG4-related disease (RD) $\geq 135 \mathrm{mg} / \mathrm{dl}$ ). Pituitary gland and/or stalk swelling was observed in six patients, and an empty sella was observed in one patient. Multiple co-existing organ involvement was observed in four of the seven patients prior to the onset of IgG4-related hypophysitis.

Conclusion: These data suggest that the prevalence of IgG4-related hypophysitis has been underestimated. We should also consider the possibility of the development of hypopituitarism/DI caused by IgG4-related hypophysitis during the clinical course of other IgG4-RDs.

\section{Introduction}

IgG4-related disease (RD) is a newly recognized clinical entity that was first proposed following the close observation of patients with autoimmune pancreatitis in 2001 (1). IgG4-RD is characterized by IgG4 ${ }^{+}$plasma cell and lymphocyte infiltration and an elevated serum IgG4 concentration. This disease involves various tissues and is associated with Mikulicz's disease, autoimmune pancreatitis, Riedel's thyroiditis, interstitial pneumonitis,
(C) 2014 European Society of Endocrinology Printed in Great Britain
Published by Bioscientifica Ltd. 
interstitial nephritis, prostatitis, lymphadenopathy, retroperitoneal fibrosis, inflammatory aortic aneurysm, and inflammatory pseudotumor (2).

Autoimmune hypophysitis, often referred to as lymphocytic hypophysitis, is defined as an inflammatory pituitary disease with an autoimmune etiology that leads to pituitary dysfunction. Primary hypophysitis is the most common form of hypophysitis and can occur in isolation or as a part of multiorgan disease that includes polyglandular autoimmune syndromes. Recently, IgG4-related hypophysitis has emerged as a part of IgG4-RD. It was initially diagnosed in a clinical setting in 2004 (3) and then by pathological examination in 2007 (4). Histopathologically, pituitary masses typically demonstrate a dense lymphoplasmacytic infiltrate among residual nests of adenohypophyseal cells and fibrosis. Immunohistochemical staining for IgG4 and $\kappa / \lambda$ light chains has revealed the presence of numerous polyclonal plasma cells in the pituitary.

Recently, Shimatsu et al. (5) have reviewed 22 patients with IgG4-related hypophysitis and reported their clinical characteristics. The majority of IgG4-related hypophysitis cases were observed in middle-aged and elderly men presenting with various degrees of hypopituitarism and diabetes insipidus (DI) and with a thickened pituitary stalk and/or mass. The pituitary structures shrank significantly after glucocorticoid therapy, even in patients treated with low doses. The presence of IgG4-RD and elevated concentrations of IgG4 before glucocorticoid therapy were the main findings that led to the diagnosis. Probably because IgG4-RD was first described in Japan (1), most of the IgG4-related hypophysitis cases that have been reported were observed among Japanese individuals (6). Leporati et al. (7), the first to report IgG4-related hypophysitis in Caucasian patients, reviewed the published literature and proposed diagnostic criteria on the basis of findings of 11 cases that were analyzed. Although the first IgG4-related hypophysitis case was described in 2004 (3), only six histologically proven cases have been reported $(3,4,7,8,9$, $10,11,12,13,14,15,16,17,18,19,20,21)$.

Because of the limited number of reported cases, the prevalence and clinical characteristics of IgG4-related hypophysitis remain incompletely defined. In this study, we screened consecutive outpatients with hypopituitarism and/or DI to estimate its prevalence. We detected seven new cases of IgG4-related hypophysitis, estimated the prevalence of IgG4-related hypophysitis among patients with hypopituitarism and/or DI, and outlined the clinical characteristics. A current review of the literature is also provided.

\section{Subjects and methods}

\section{Subjects}

We screened 170 consecutive outpatients with hypopituitarism and/or central DI at Kobe University Hospital between 2011 and 2012. For the diagnosis of adult growth hormone deficiency, each patient was subjected to an insulin tolerance test or growth hormone-releasing hormone test (22). The hypothalamic-pituitaryadrenal axis, hypothalamic-pituitary-thyroid axis, and hypothalamic-pituitary-gonadal axis were evaluated as described previously (23). Patients who had already been diagnosed with known causes were excluded (Fig. 1). Additionally, patients with isolated adrenocorticotropic hormone (ACTH) deficiency were excluded and analyzed separately. Isolated ACTH deficiency was defined as secondary adrenal insufficiency with low or absent cortisol production and normal secretion of pituitary hormones other than ACTH (24). Among the remaining patients with hypophysitis or without known causes of hypopituitarism and/or central DI, we tried to identify criteria suggesting IgG4-related hypophysitis: i) serum IgG4 concentrations $\geq 135 \mathrm{mg} / \mathrm{dl}$; ii) involvement of other organs observed in IgG4-RD; and/or iii) infiltration of IgG4-positive plasma cells in the involved tissues $(1,2,5$, 25, 26) (Fig. 1). Hypophysitis was diagnosed based on the magnetic resonance imaging (MRI) findings with an enlargement of the anterior pituitary and/or stalk with extension of the thickened stalk up toward the hypothalamus with homogeneous gadolinium enhancement, pituitary biopsy, and clinical course as described previously (27).

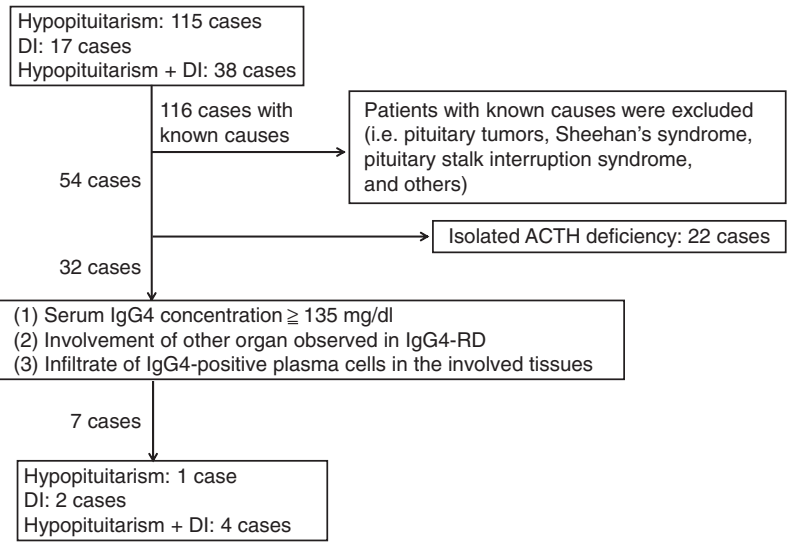

Figure 1

Study design of the screening for IgG4-related hypophysitis. $\mathrm{DI}$, diabetes insipidus. 


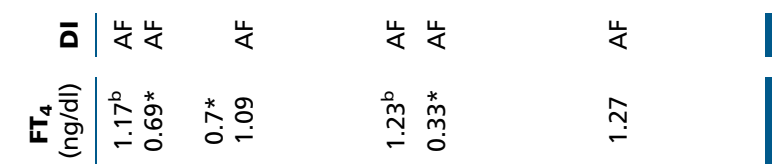

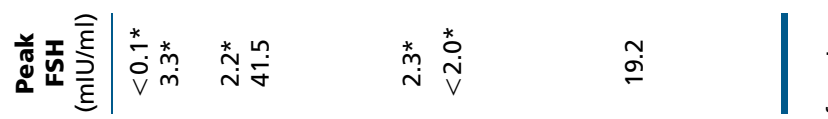

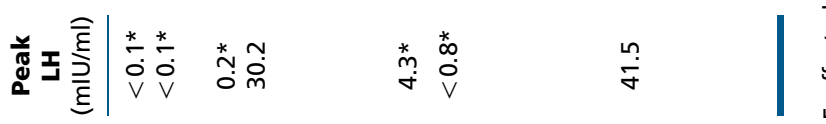

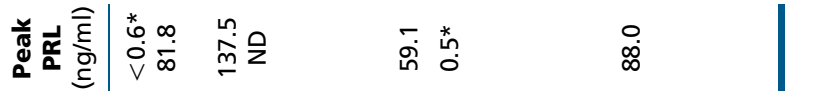

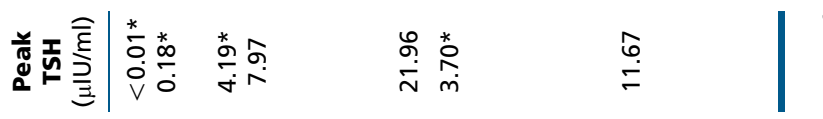

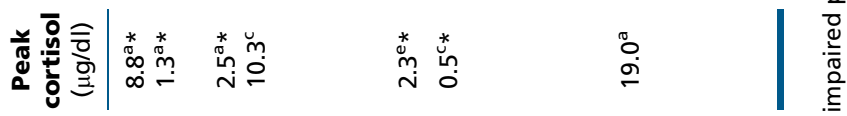

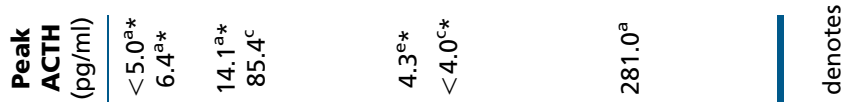

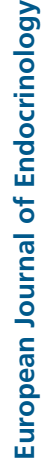

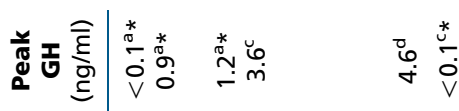

$\stackrel{\sim}{\sim}$

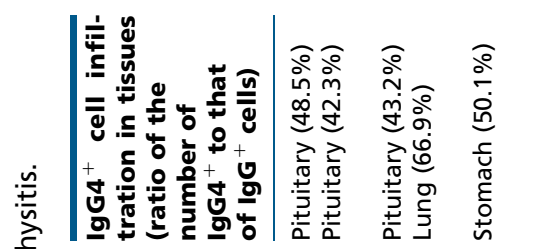
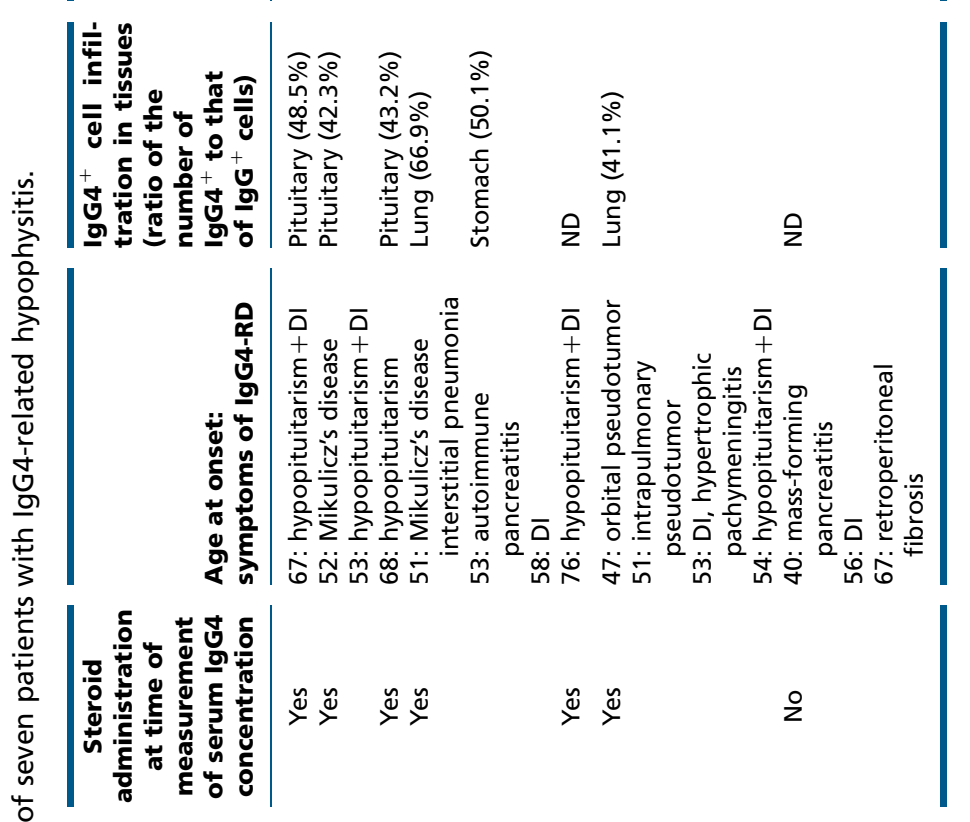

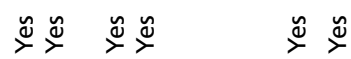

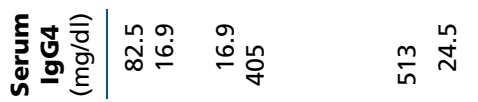

范范范

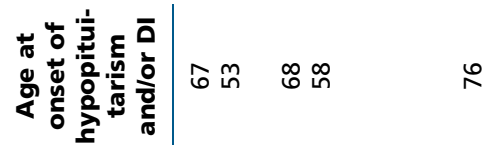

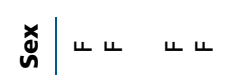

ํํํ

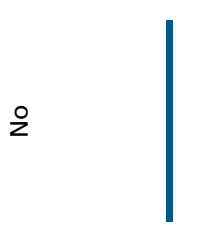

$\frac{\mathscr{y}}{4}$

$\stackrel{\overbrace{}}{\sim}$

$\stackrel{1}{2}$

$\Sigma \Sigma$

$\circ$

$$
\Sigma
$$$$
\Sigma
$$

Ln 6

$\wedge$ 


\section{Methods}

Measurement of serum IgG4 concentrations > Serum IgG4 concentrations were measured using IgG4 (BS-NIA) kits (The Binding Site, Birmingham, UK). The normal value for IgG4 concentrations ranges from 5 to $105 \mathrm{mg} / \mathrm{dl}$. The criterion of IgG4 levels in IgG4-RD was defined as more than $135 \mathrm{mg} / \mathrm{dl}(28)$.

Immunohistochemistry $\bullet \mathrm{IgG}$ and IgG4 immunostaining was performed using a BenchMark XT IHC/ISH staining module (Ventana Medical Systems, Tucson, AZ, USA) according to the manufacturer's instructions (29). Polyclonal rabbit antibody against human IgG (Diagnostic BioSystems, Fremont, CA, USA) and mouse MAB against human IgG4 (Zymed Laboratories, South San Francisco, CA, USA) were used as primary antibodies. Images were obtained with a BZ-8100 microscope (Keyence, Osaka, Japan).

\section{Results}

Among the 170 patients screened, 116 patients who had already been diagnosed with obvious known causes including pituitary tumors demonstrated by histology at the time of surgery or biopsy, Sheehan's syndrome, pituitary stalk interruption syndrome, congenital abnormalities, Langerhan's cell histiocytosis, and Hand-SchullerChristian disease were excluded. Additionally, 22 patients with isolated ACTH deficiency were analyzed and were found to not meet the criteria of IgG4-related hypophysitis. Among the remaining 32 patients (12 men and 20 women; age $53.0 \pm 17.0$ years), we identified criteria for suggesting IgG4-related hypophysitis. This group included 13 patients presenting with hypopituitarism, 12 presenting with DI, and seven presenting with both hypopituitarism and DI. Autoimmune pancreatitis, retroperitoneal fibrosis, Mikulicz's disease, interstitial pneumonia, orbital pseudotumor, intrapulmonary pseudotumor,

Before corticosteroid administration

Case 1

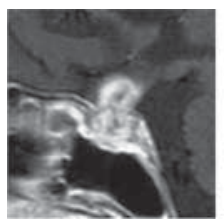

Enlargement of anterior pituitary

Swelling of pituitary stalk

Disappearance of high signal of posterior pituitary

After corticosteroid administration
Case 2
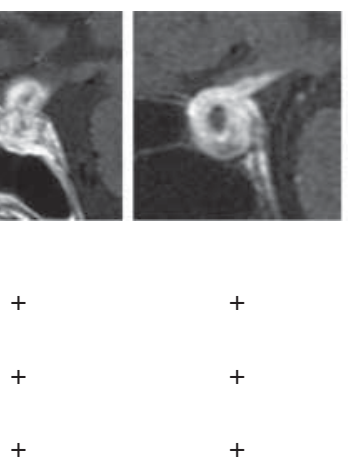

Case 3

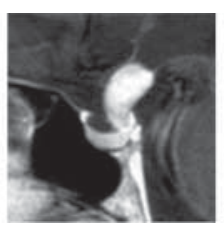

$+$

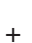

$+$

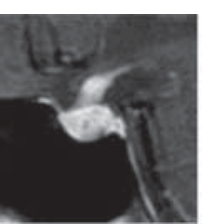

$+$

$+$

$+$
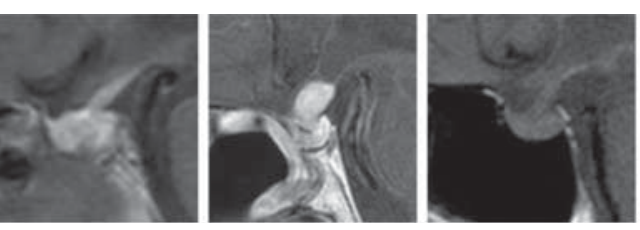

Case 5

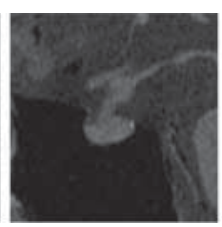

$+$

$+$

$+$

$+$

Steroid administration (-)

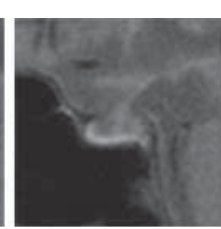

\section{Figure 2}

Pituitary magnetic resonance images before and after corticosteroid administration (gadolinium-enhanced T1-weighted images for cases 1-4 and T1-weighted images for cases 5 and 7). Images for cases 1, 2, 4, and 5 revealed an enlargement of the pituitary gland. Images for case 5 after corticosteroid administration and those for case 7 revealed an empty sella. Images for cases 1, 3, 4, and 5 showed a thickened pituitary stalk. Images for case 1 revealed thickened dura mater. Disappearance of high signal of posterior pituitary was evaluated in T1-weighted plain image. 
and hypertrophic pachymeningitis were observed in four patients, indicating the involvement of other organs (Table 1). Pituitary MRI was performed in 31 of the 32 cases; of these, four cases showed swelling of the pituitary gland, six showed enlargement of the pituitary stalk, eight showed both swelling of the pituitary gland and enlargement of the pituitary stalk, six showed pituitary atrophy including an empty sella, and three exhibited normal morphology. Among the 32 patients, nine who showed normal or atrophic pituitary were excluded and 23 were diagnosed with hypophysitis or with presumed hypophysitis when biopsy was not performed. The disappearance of high signal intensity of the posterior lobe on T1-weighted MRI was observed in 18 cases. In all, seven cases (three men and four women) met the criteria for the diagnosis of IgG4-related hypophysitis. The clinical history of each IgG4-related hypophysitis case is outlined below and in Table 1, and MRI findings are shown in Fig. 2.

\section{Case 1}

A 67-year-old woman developed sudden-onset headache and polyuria. Endocrinological examination revealed hypopituitarism and DI. There was no co-existing organ involvement.

\section{Case 2}

A 52-year-old woman was diagnosed with Mikulicz's disease. She developed hypopituitarism and DI the following year. Enlargement of the pituitary gland resolved after corticosteroid administration (Fig. 2).

\section{Case 3}

A 68-year-old woman presented with general malaise and vomiting and was diagnosed with hypophysitis (Fig. 2 and Table 1). No co-existing organ involvement was diagnosed.

\section{Case 4}

A 58-year-old woman with Mikulicz's disease, interstitial pneumonia, and autoimmune pancreatitis developed polyuria and thirst and was diagnosed with DI after 7 years of initial IgG4-RD.

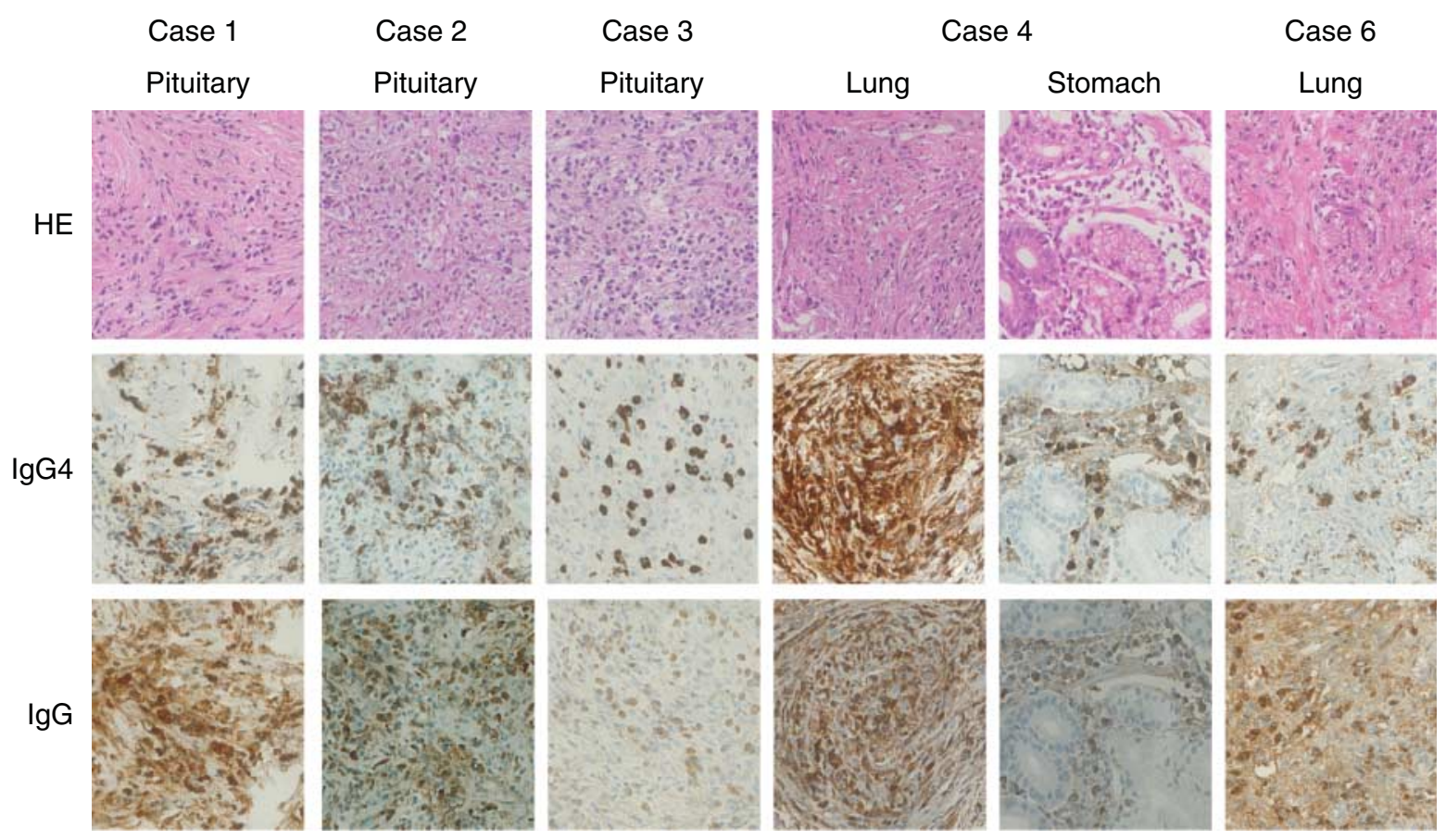

\section{Figure 3}

Histopathology of the biopsy specimens obtained from the pituitary gland (cases 1-3), lung (cases 4 and 6), stomach (case 5), and pancreas (case 7). The results of hematoxylin and eosin staining and immunohistochemical analysis of IgG4 and IgG are shown ( $\times 400$ magnification). 

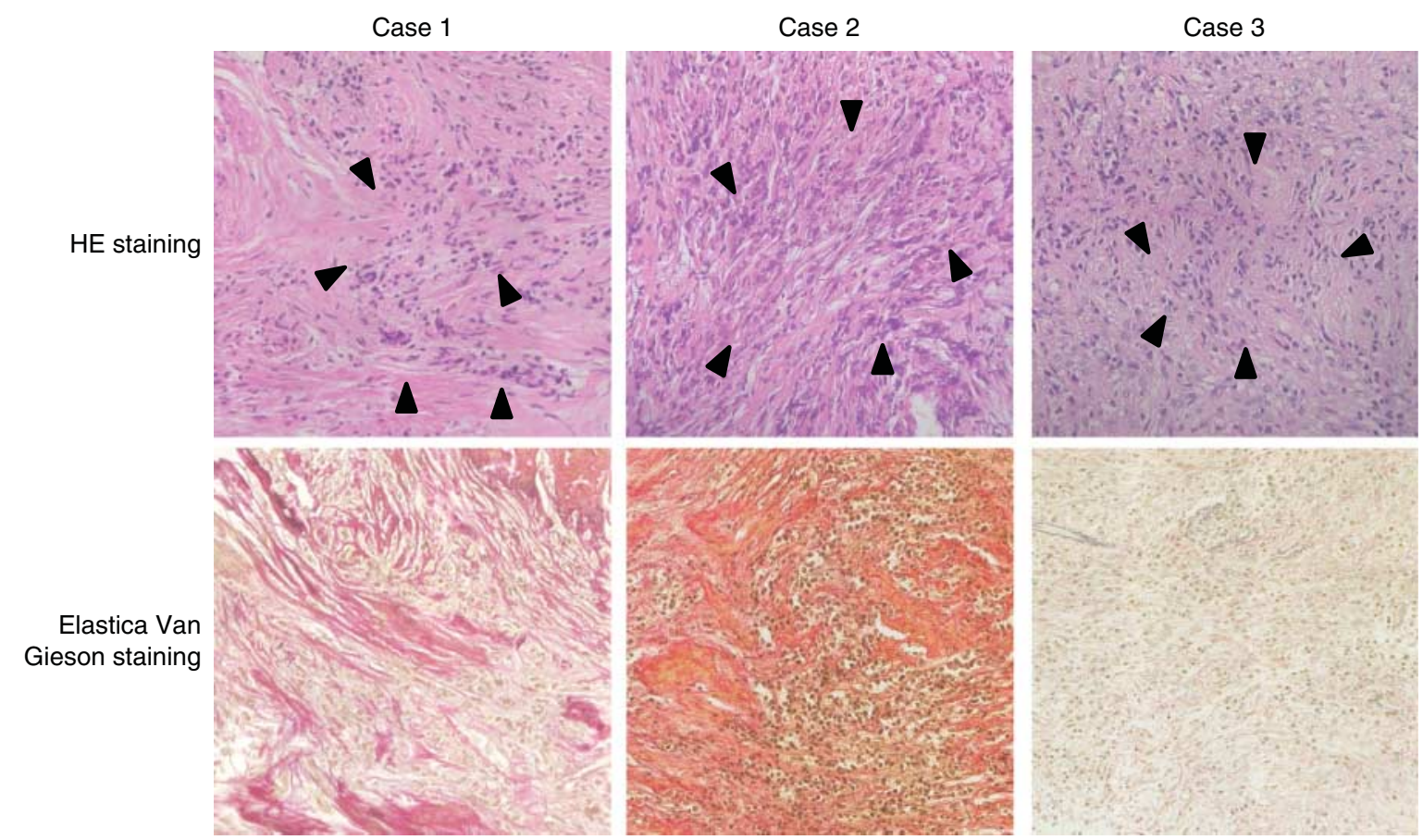

\section{Figure 4}

Histopathological features of the pituitary specimens.

The results of hematoxylin and eosin staining and Elastica van Gieson (EVG) staining are shown (×200 magnification).

\section{Case 5}

A 76-year-old man developed loss of consciousness due to hyponatremia, hypoglycemia, and polyuria. Endocrinological examination revealed the presence of hypopituitarism and DI (Table 1). After 4 years of replacement therapy, the anterior pituitary atrophied and the swelling of the stalk improved (Fig. 2).

\section{Case 6}

The details of this case have been reported previously (30); however, IgG4-related hypophysitis was not diagnosed at that time. In brief, a 53-year-old man with orbital pseudotumor and pulmonary pseudotumor developed polyuria and polydipsia and was subsequently diagnosed with hypopituitarism and DI. Pituitary MRI revealed a swelling of the pituitary stalk and disappearance of the high signal intensity of the posterior lobe (30). IgG4related hypophysitis was diagnosed by the immunohistochemical analysis of lung biopsy specimen (Fig. 3) this time.
Arrowheads indicate the typical 'storiform pattern' observed in the pituitary tissue. Any features of obliterative phlebitis by EVG staining could not be found.

\section{Case 7}

A 40-year-old man was diagnosed with mass-forming pancreatitis. At the age of 56 years, the patient developed polyuria and was diagnosed with DI. At the age of 67 years, abdominal computed tomography (CT) revealed retroperitoneal fibrosis.

Three cases were diagnosed by the analysis of pituitary biopsy specimens (cases 1-3; Fig. 4). Three patients exhibited elevated serum IgG4 concentrations (cases 4, 5 , and 7). One patient was diagnosed with a presence of morphological abnormalities of the pituitary with impaired function and co-presence of IgG4-RD (case 6). Pituitary dysfunction did not improve after corticosteroid administration in any case. The ages (mean \pm s.D.) at the time of the development of IgG4-RD and hypophysitis were $57.3 \pm 11.3$ and $61.8 \pm 8.8$ years respectively. The serum IgG4 concentration (mean \pm s.D.) was $191.1 \pm$ $78.3 \mathrm{mg} / \mathrm{dl}$. Pituitary MRI revealed an enlargement of the pituitary gland and thickened pituitary stalk in six of the seven cases, and one case (case 7) demonstrated an empty sella. Thickened dura mater was detected in 
cases 1 and 6 (Fig. 2). Accordingly, IgG4-related hypophysitis was detected in 30\% (seven of 23 cases) of hypophysitis cases, $22 \%$ (seven of 32 cases) of hypopituitarism/DI cases with unknown causes, and 4\% (seven of 170 cases) of all cases of hypopituitarism/DI (Fig. 5). Furthermore, IgG4-related hypophysitis was diagnosed in $8 \%$ of hypopituitarism cases (one of 13 patients), $17 \%$ of DI cases (two of 12 patients), and $57 \%$ of hypopituitarism and DI cases (four of seven patients) that involved impaired anterior or posterior pituitary function with an unknown cause.

\section{Discussion}

Herein, we report seven new cases of IgG4-related hypophysitis. Based on the criteria defined by Leporati et al. (7), a diagnosis of IgG4-related hypophysitis was made in cases 1-6. IgG4/IgG-positive cells were not detected upon immunostaining of the pancreatic specimens in case 7 (data not shown). This case was classified as a 'probable' case of IgG4-RD based on the diagnostic criteria because of the negative staining results for IgG4 in the pancreatic specimens, although serum IgG4 concentration was elevated (28). Three of the seven patients were men. All the cases involved middle-aged and elderly patients presenting with hypopituitarism and/or DI. Six of the seven cases showed a thickened pituitary stalk and/or pituitary mass observed using MRI. Except in case 3, these abnormal structures shrank significantly in response to glucocorticoid therapy even in patients treated with a low dose. However, none of the patients showed a restored pituitary function after steroid therapy. Five patients presented with IgG4-RD attributable to the involvement of other organs, and three patients showed an elevated IgG4 concentration. Four patients who exhibited normal serum IgG4 concentrations were histologically confirmed.

A summary of the reported cases of IgG4-related hypophysitis, including the present cases, is given in Table $2(3,4,7,8,9,10,11,12,13,14,15,16,17,18,19$, $20,21,30,31,32,33,34)$. To our knowledge, there have been only 22 reports with 29 cases (22 men and seven women) including the present cases that met the criteria for diagnosis, suggesting that IgG4-related hypophysitis may be extremely rare. The ages (mean \pm s.D.) at the time of the onset of IgG4-RD and hypophysitis were $60.6 \pm 11.2$ and $63.3 \pm 10.4$ years respectively. The age distribution at the time of the onset of hypophysitis was as follows: three patients were in their 40s, seven in their 50s, nine in their $60 \mathrm{~s}$, and ten in their 70s. Previous studies have reported that the median ages at the time of the onset of AIP and

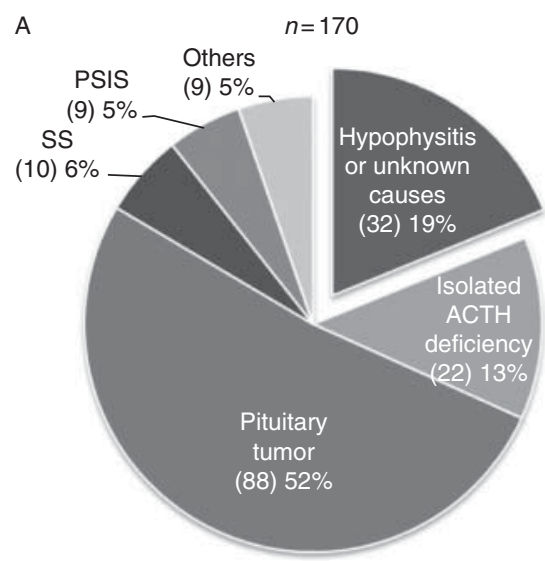

B

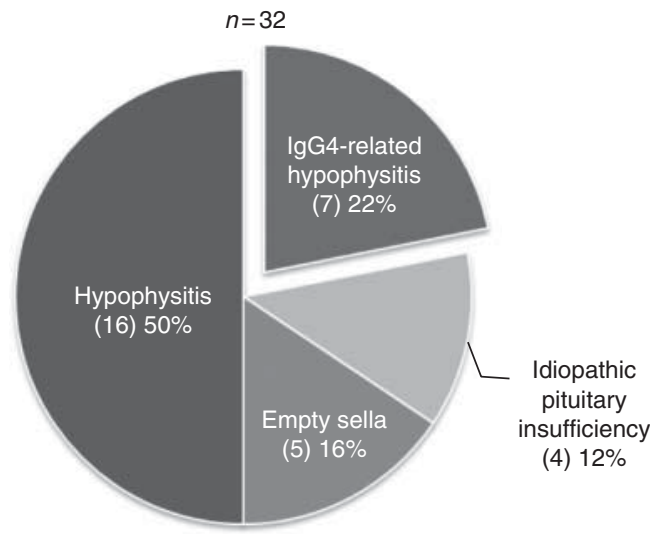

C

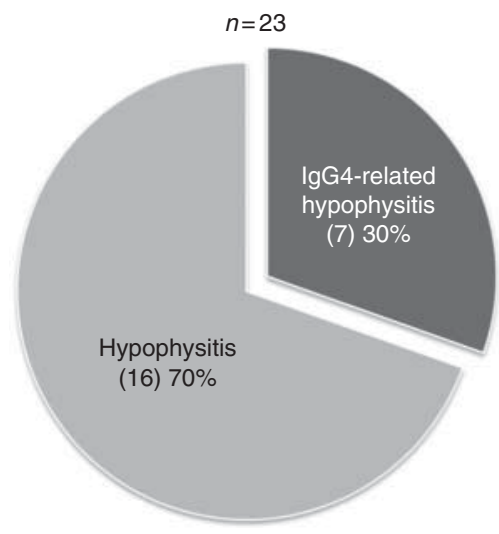

Figure 5

(A) Causes of hypopituitarism and/or DI in all patients. PSIS, pituitary stalk interruption syndrome; SS, Sheehan's syndrome. (B) Prevalence of IgG4-related hypophysitis in patients with general hypophysitis or unknown causes. (C) Prevalence of IgG4-related hypophysitis in patients with hypophysitis. Number of patients is given within parentheses. 
||

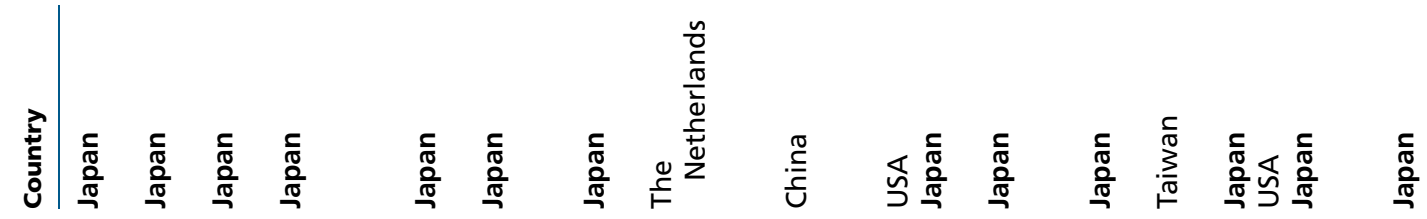

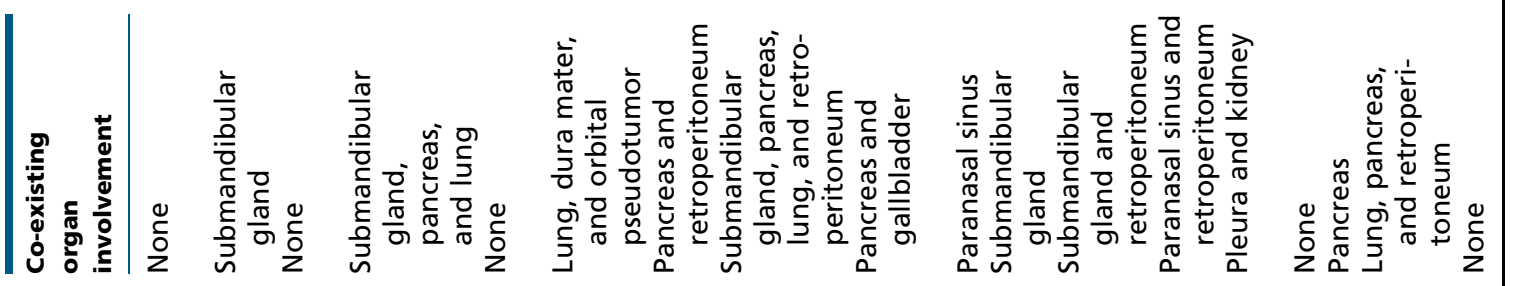

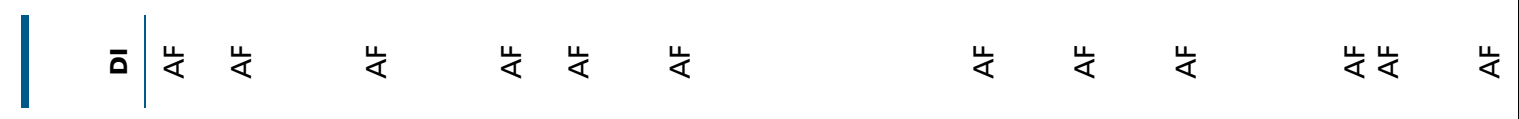
|y

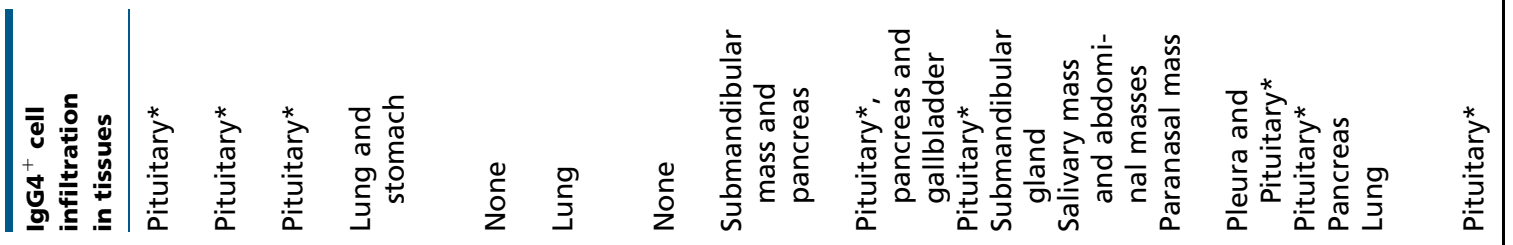

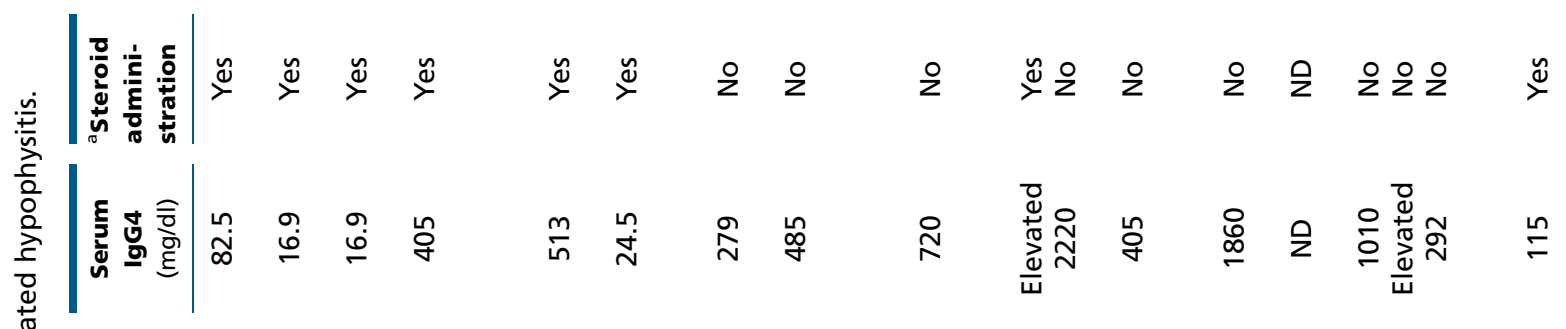
|

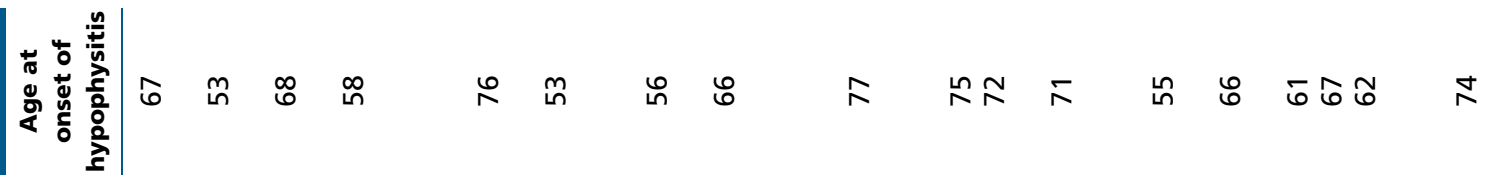

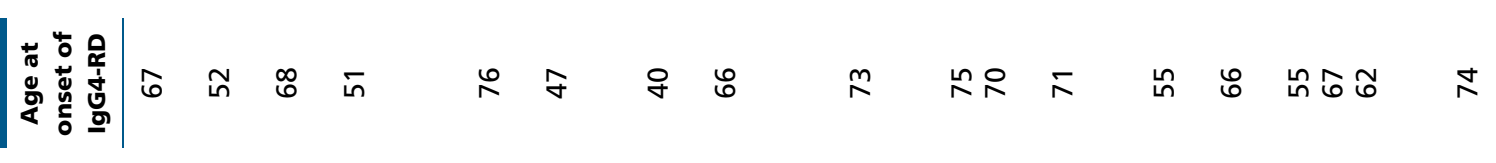

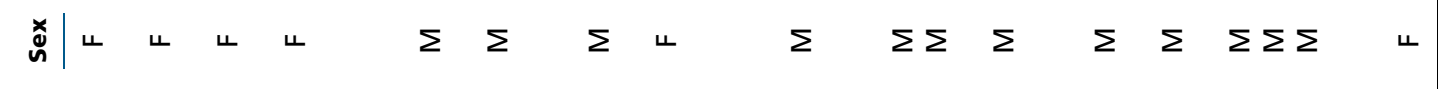

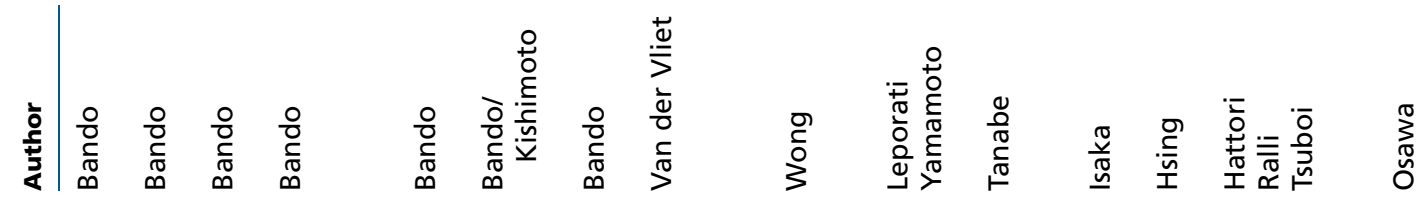
敦| 


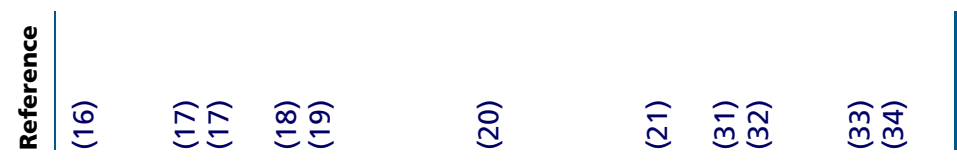

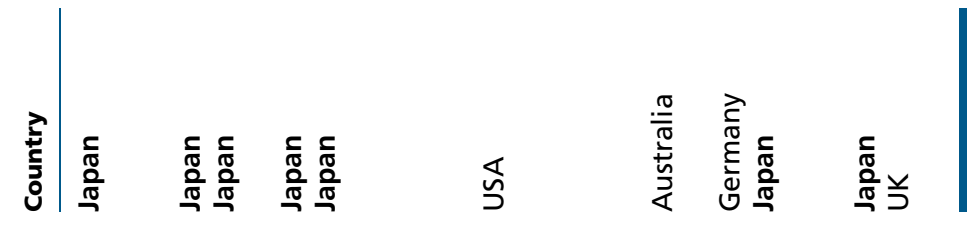

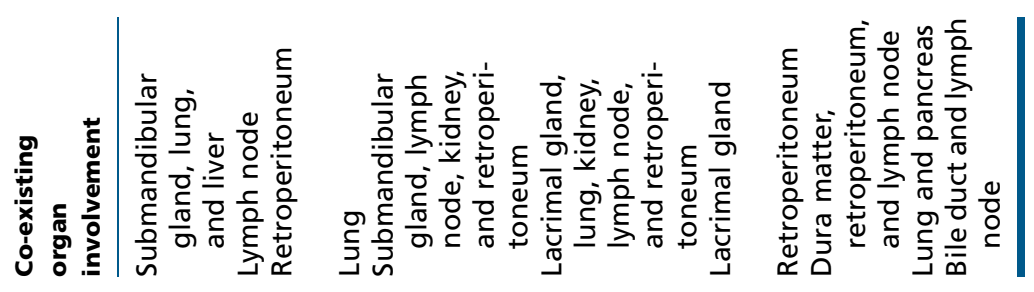

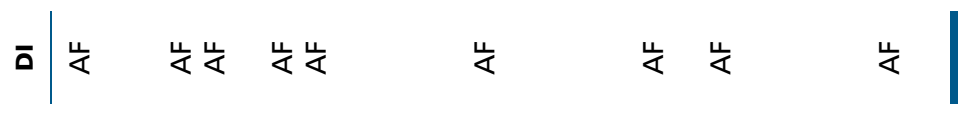

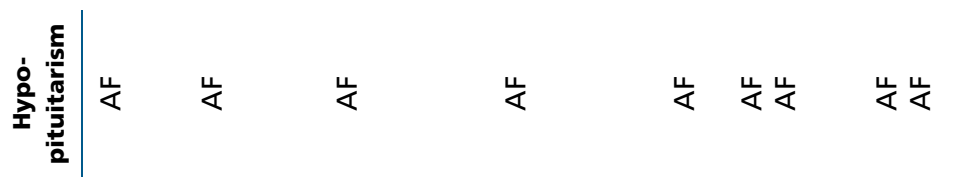

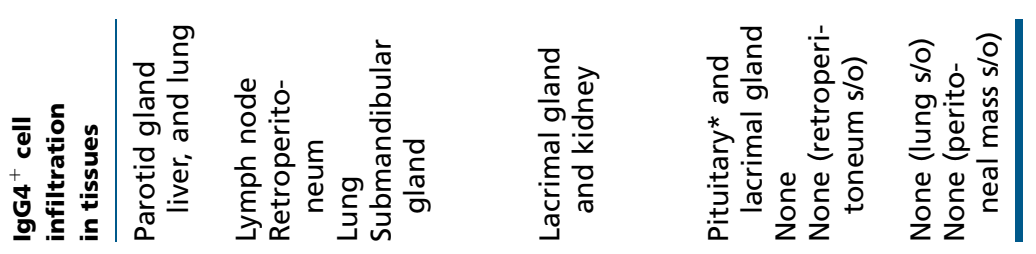

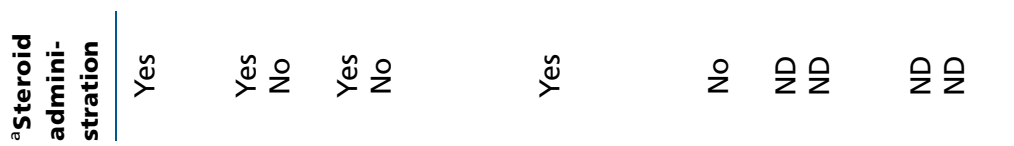

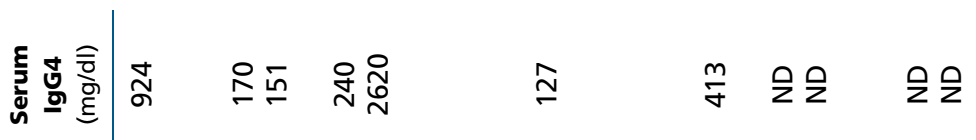

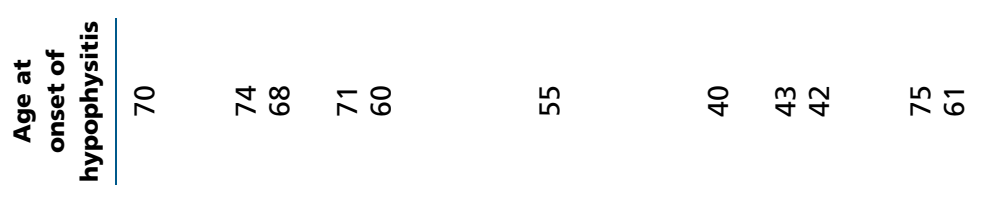

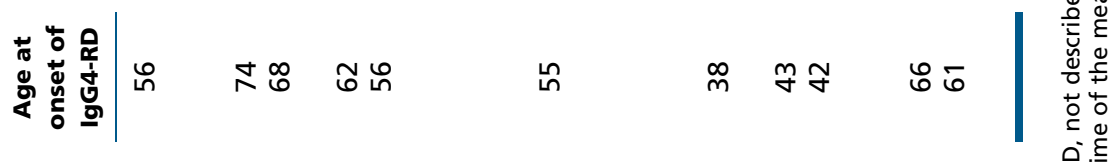

$\stackrel{\times}{\circ}|\Sigma \quad 4 \Sigma \Sigma \Sigma \quad \Sigma \quad \Sigma \quad \Sigma \Sigma \quad \Sigma \Sigma|$

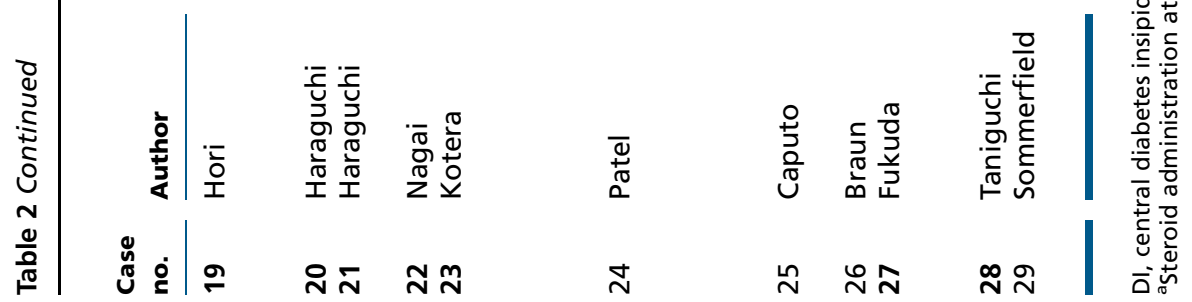


IgG4-related hypophysitis are 58 and 64 years respectively $(2,5)$ and the male-to-female ratios are 2.9:1 and 21:1 respectively $(5,35)$. Hypopituitarism was observed in 24 cases (83\%) and DI was observed in 21 cases (72\%). Seventeen cases presented with both hypopituitarism and DI (59\%). An elevation of serum IgG4 concentration was observed in 18 of the 24 cases (75\%). Serum IgG4 concentration (mean \pm s.D.) measured prior to corticosteroid administration was $950.5 \pm 872.3 \mathrm{mg} / \mathrm{dl}$ in 11 cases, which was much higher than that for all cases $(595.0 \pm$ $729.1 \mathrm{mg} / \mathrm{dl})$, suggesting that glucocorticoid therapy decreases serum IgG4 concentrations. Caputo et al. (21) reported a case in which serum IgG4 concentration was reduced after prednisolone administration, which again indicates the importance of measuring IgG4 concentrations before steroid replacement therapy. The involvement of various organs was noted throughout the clinical course, with retroperitoneal fibrosis being the most common form (38\%), followed by interstitial pneumonia (eight cases, 28\%), Mikulicz's disease and AIP (seven cases, $24 \%$ ), and lymph node swelling (five cases, 18\%). Only four patients (14\%) solely exhibited hypophysitis. In most cases, organ involvement occurred prior to the onset of hypophysitis; however, retroperitoneal fibrosis developed after the onset of hypophysitis in two cases. Thus far, most of the patients have been reported in Japan. Although the precise reason is unknown, this may be associated with the racial difference, the spread of awareness of IgG4-RD, and easy accessibility to MRI in Japan.

In this study, IgG4-related hypophysitis was detected in 30\% (seven of 23 cases) of hypophysitis cases, $22 \%$ (seven of 32 cases) of hypopituitarism/DI cases with unknown causes, and 4\% (seven of 170 cases) of all cases of hypopituitarism/DI cases respectively. Additionally, these data suggest that the prevalence of IgG4-related hypophysitis has been underestimated. One of the reasons for the underestimation is that the elevated serum IgG4 concentration is easily normalized even by a physiological dose of steroids. Indeed, we diagnosed four cases with normal serum IgG4 concentrations by examining biopsy specimens of the pituitary or other involved tissues including the lung and stomach. In this regard, it is important to check the involvement of other organs carefully. Importantly, four patients presented other IgG4RDs prior to the onset of IgG4-related hypophysitis. Considering the fact that the number of IgG-RD cases reported, such as autoimmune pancreatitis, has increased owing to the increased awareness of the condition (25), it is important to follow up these cases by considering a possibility of pituitary dysfunction caused by IgG4-related hypophysitis. Although the distribution of the causes of pituitary insufficiency in this study (Fig. 5A) may differ from those in other series, in which tumors were more predominant $(23,36)$, this may be reflected by the fact that our division belongs to a department of internal medicine. There may also be a selection bias for the screened patients because of the specialty outpatient clinic in our university hospital. Nevertheless, the substantial prevalence of IgG4related hypophysitis should be emphasized.

We screened patients with isolated ACTH deficiency because autoimmunity has been suggested as its etiology and a relationship with hypophysitis has been suggested (24). In our series, none of the patients with isolated ACTH deficiency were diagnosed with IgG4-related hypophysitis. This may be explained by a possible decrease in serum IgG4 concentrations by steroid replacement therapy; however, we could not identify the involvement of any other organ in these 22 cases of isolated ACTH deficiency, suggesting that isolated ACTH deficiency may belong to a clinical spectrum different from IgG4-related hypophysitis.

The MRI findings in most patients with IgG4-related hypophysitis were similar to those observed in cases of general hypophysitis, such as an enlargement of the anterior pituitary and/or stalk. Case 2 exhibited a cystic formation in the enlarged anterior pituitary. Case 7 exhibited an empty sella and case 5 exhibited an empty sella after steroid replacement therapy. Although the final condition of IgG4-related hypophysitis has not been reported, Gao et al. (37) reported that an empty sella is detected after a long period of treatment with glucocorticoids in cases of autoimmune hypophysitis. Furthermore, development of the pituitary from the expanded state to an empty sella has been reported in a mouse model of autoimmune hypophysitis (38). These data suggest that at least in some cases, IgG4-related hypophysitis results in an empty sella after a stage of swelling.

Immunohistochemical analysis of the pituitary tissue and the detection of the presence of $\operatorname{IgG} 4^{+}$lymphocytes are essential for the definitive diagnosis of IgG4-related hypophysitis $(4,5,7,25)$. In three cases, we were able to make a diagnosis by pituitary biopsy despite normal serum IgG4 concentrations. This discrepancy between the tissue findings and serum concentrations of IgG4 may be explained by glucocorticoid therapy, even when administered in physiological doses. In light of these results, it is recommended that in patients with a pituitary mass due to unknown causes, serum IgG4 concentration be measured at least once before glucocorticoid treatment. In addition, even after glucocorticoid treatment, 
immunohistochemical analysis of tissue specimens for IgG4 staining is a useful method for diagnosing IgG4related hypophysitis. In particular, we found that the gastric polypoid lesion observed in case 4 was also positive for IgG4 staining, suggesting that immunochemical staining of various tissue lesions is worth performing in patients with hypopituitarism and/or DI exhibiting a pituitary mass (39).

Notably, histological analysis revealed a characteristic storiform fibrosis (i.e. matted and irregularly whorled) in all three pituitary tissue specimens, which is a key morphological feature of IgG4-RD. Other morphological features include obliterative phlebitis $(2,40,41)$. Although these characteristics are generally common in autoimmune pancreatitis, they have not been reported in IgG4-related hypophysitis. We could not detect any features of obliterative phlebitis in the pituitary lesions; the reason is unknown, but the unique portal system in the pituitary may affect these characteristics. The presence of a storiform pattern may be an important clue for diagnosing IgG4-related hypophysitis as well as the immunostaining for IgG4 plasma cells.

In conclusion, this study reveals a substantial prevalence of IgG4-related hypophysitis in patients with hypopituitarism/DI. IgG4-RD may cause hypopituitarism/ DI associated with IgG4-related hypophysitis: hence, patients with IgG4-RD should be carefully followed up. Additionally, IgG4-RD should be considered in all cases presenting with hypophysitis, especially as it responds well to treatment and also as this is a systemic disease.

\section{Declaration of interest}

The authors declare that there is no conflict of interest that could be perceived as prejudicing the impartiality of the research reported.

\section{Funding}

This work was supported in part by a Grant-in-Aid for Scientific Research from the Japanese Ministry of Education, Culture, Sports, Science, and Technology 23659477, 23591354, and 22591012, Grants-in-Aid for Scientific Research (research on hypothalamic-hypophyseal disorders) from the Ministry of Health, Labor, and Welfare, Japan, Daiichi-Sankyo Foundation of Life Science, and the Naito Foundation.

\section{Author contribution statement}

$\mathrm{H}$ Bando drafted the manuscript and analyzed and interpreted the data. $\mathrm{G}$ Iguchi was responsible for the conception and design of the study. M Taniguchi and E Kohmura collected pituitary samples. H Fukuoka, M Yamamoto, R Matsumoto, K Suda, H Nishizawa, and M Takahashi collected and assembled the data. $Y$ Takahashi was responsible for the critical revision of the article for important intellectual content.

\section{Acknowledgements}

The authors are grateful to Dr T Nakai for discussion and C Ogata and $\mathrm{K}$ Imura for their excellent technical assistance.

\section{References}

1 Hamano H, Kawa S, Horiuchi A, Unno H, Furuya N, Akamatsu T, Fukushima M, Nikaido T, Nakayama K, Usuda N et al. High serum IgG4 concentrations in patients with sclerosing pancreatitis. New England Journal of Medicine 2001344 732-738. (doi:10.1056/ NEJM200103083441005)

2 Umehara H, Okazaki K, Masaki Y, Kawano M, Yamamoto M, Saeki T, Matsui S, Sumida T, Mimori T, Tanaka Y et al. A novel clinical entity, IgG4-related disease (IgG4RD): general concept and details. Modern Rheumatology 201222 1-14. (doi:10.1007/s10165-011-0508-6)

3 van der Vliet HJ \& Perenboom RM. Multiple pseudotumors in IgG4associated multifocal systemic fibrosis. Annals of Internal Medicine 2004 141 896-897. (doi:10.7326/0003-4819-141-11-200412070-00033)

4 Wong S, Lam WY, Wong WK \& Lee KC. Hypophysitis presented as inflammatory pseudotumor in immunoglobulin G4-related systemic disease. Human Pathology 200738 1720-1723. (doi:10.1016/j.humpath. 2007.06.011)

5 Shimatsu A, Oki Y, Fujisawa I \& Sano T. Pituitary and stalk lesions (infundibulo-hypophysitis) associated with immunoglobulin G4related systemic disease: an emerging clinical entity. Endocrine Journal 200956 1033-1041. (doi:10.1507/endocrj.K09E-277)

6 Caturegli P \& Iwama S. From Japan with love: another tessera in the hypophysitis mosaic. Journal of Clinical Endocrinology and Metabolism 201398 1865-1868. (doi:10.1210/jc.2013-1912)

7 Leporati P, Landek-Salgado MA, Lupi I, Chiovato L \& Caturegli P. IgG4-related hypophysitis: a new addition to the hypophysitis spectrum. Journal of Clinical Endocrinology and Metabolism 201196 1971-1980. (doi:10.1210/jc.2010-2970)

8 Yamamoto M, Takahashi H, Ohara M, Suzuki C, Naishiro Y, Yamamoto H, Shinomura Y \& Imai K. A case of Mikulicz's disease (IgG4-related plasmacytic disease) complicated by autoimmune hypophysitis. Scandinavian Journal of Rheumatology 200635 410-411. (doi:10.1080/03009740600758110)

9 Tanabe T, Tsushima K, Yasuo M, Urushihata K, Hanaoka M, Koizumi T Fujimoto K, Kubo K, Uehara T, Shigematsu S et al. IgG4-associated multifocal systemic fibrosis complicating sclerosing sialadenitis, hypophysitis, and retroperitoneal fibrosis, but lacking pancreatic involvement. Internal Medicine 200645 1243-1247. (doi:10.2169/ internalmedicine.45.1759)

10 Isaka Y, Yoshioka K, Nishio M, Yamagami K, Konishi Y, Inoue T, Hirano A, Hosoi M \& Imanishi M. A case of IgG4-related multifocal fibrosclerosis complicated by central diabetes insipidus. Endocrine Journal 200855 723-728. (doi:10.1507/endocrj.K08E-034)

11 Hsing MT, Hsu HT, Cheng CY \& Chen CM. IgG4-related hypophysitis presenting as a pituitary adenoma with systemic disease. Asian Journal of Surgery 201336 93-97. (doi:10.1016/j.asjsur.2012.04.013)

12 Hattori Y, Tahara S, Ishii Y, Kitamura T, Inomoto C, Osamura RY, Teramoto A \& Morita A. A case of IgG4-related hypophysitis without pituitary insufficiency. Journal of Clinical Endocrinology and Metabolism 201398 1808-1811. (doi:10.1210/jc.2013-1088)

13 Ralli S, Lin J \& Farrell J. Autoimmune pancreatitis. New England Journal of Medicine 2007356 1586. (doi:10.1056/NEJMc070069)

14 Tsuboi H, Inokuma S, Setoguchi K, Shuji S, Hagino N, Tanaka Y, Yoshida N, Hishima T \& Kamisawa T. Inflammatory pseudotumors in multiple organs associated with elevated serum IgG4 level: recovery by only a small replacement dose of steroid. Internal Medicine 200847 1139-1142. (doi:10.2169/internalmedicine.47.0887)

15 Osawa S, Ogawa Y, Watanabe M \& Tominaga T. Hypophysitis presenting with atypical rapid deterioration: with special reference to 
immunoglobulin G4-related disease-case report. Neurologia MedicoChirurgica 200949 622-625. (doi:10.2176/nmc.49.622)

16 Hori M, Makita N, Andoh T, Takiyama H, Yajima Y, Sakatani T, Fukumoto S, Iiri T \& Fujita T. Long-term clinical course of IgG4-related systemic disease accompanied by hypophysitis. Endocrine Journal 2010 57 485-492. (doi:10.1507/endocrj.K09E-356)

17 Haraguchi A, Era A, Yasui J, Ando T, Ueki I, Horie I, Imaizumi M, Usa T, Abe K, Origuchi T et al. Putative IgG4-related pituitary disease with hypopituitarism and/or diabetes insipidus accompanied with elevated serum levels of IgG4. Endocrine Journal 201057 719-725. (doi:10.1507/ endocrj.K10E-030)

18 Nagai K, Hara Y, Shinkai M, Goto H, Hoshino M, Watanabe K, Yamaguchi N, Kawana A, Ishigatsubo Y \& Kaneko T. A case of IgG4related disease with deterioration in pulmonary and pituitary involvements during a 10-year clinical course of inflammatory pseudotumor. Nihon Kokyuki Gakkai Zasshi 201149 922-928.

19 Kotera N, Isogawa A, Uchida L, Ishimoto Y, Tanaka M, Tanaka S, Kishi S, Mise N, Sugimoto T \& Shiba T. Case report: IgG4-related hypophysitis presenting with secondary adrenal insufficiency and central diabetes insipidus in a type 1 diabetes patient. Nihon Naika Gakkai Zasshi 2011 100 1044-1047. (doi:10.2169/naika.100.1044)

20 Patel SM \& Szostek JH. IgG4-related systemic disease in a Native American man. Internal Medicine 201150 931-934. (doi:10.2169/ internalmedicine.50.4593)

21 Caputo C, Bazargan A, McKelvie PA, Sutherland T, Su CS \& Inder WJ. Hypophysitis due to IgG4-related disease responding to treatment with azathioprine: an alternative to corticosteroid therapy. Pituitary 2014. In press. (doi:10.1007/s11102-013-0498-9)

22 Molitch ME, Clemmons DR, Malozowski S, Merriam GR, Shalet SM, Vance ML, Endocrine Society's Clinical Guidelines Subcommittee \& Stephens PA. Evaluation and treatment of adult growth hormone deficiency: an Endocrine Society Clinical Practice Guideline. Journal of Clinical Endocrinology and Metabolism 200691 1621-1634. (doi:10.1210/ jc.2005-2227)

23 Tomlinson JW, Holden N, Hills RK, Wheatley K, Clayton RN, Bates AS, Sheppard MC \& Stewart PM. Association between premature mortality and hypopituitarism. West Midlands Prospective Hypopituitary Study Group. Lancet 2001357 425-431. (doi:10.1016/S01406736(00)04006-X)

24 Andrioli M, Pecori Giraldi F \& Cavagnini F. Isolated corticotrophin deficiency. Pituitary 20069 289-295. (doi:10.1007/s11102-006-0408-5)

25 Stone JH, Zen Y \& Deshpande V. IgG4-related disease. New England Journal of Medicine 2012366 539-551. (doi:10.1056/NEJMra1104650)

26 Deshpande V, Zen Y, Chan JK, Yi EE, Sato Y, Yoshino T, Klöppel G, Heathcote JG, Khosroshahi A, Ferry JA et al. Consensus statement on the pathology of IgG4-related disease. Modern Pathology 201225 1181-1192. (doi:10.1038/modpathol.2012.72)

27 Laws ER, Vance ML \& Jane JA Jr. Hypophysitis. Pituitary 20069 331-333. (doi:10.1007/s11102-006-0415-6)

28 Umehara H, Okazaki K, Masaki Y, Kawano M, Yamamoto M, Saeki T, Matsui S, Yoshino T, Nakamura S, Kawa S et al. Comprehensive diagnostic criteria for IgG4-related disease (IgG4-RD), 2011. Modern Rheumatology 201222 21-30. (doi:10.1007/s10165-011-0571-z)
29 Zen Y, Inoue D, Kitao A, Onodera M, Abo H, Miyayama S, Gabata T, Matsui $\mathrm{O} \&$ Nakanuma Y. IgG4-related lung and pleural disease: a clinicopathologic study of 21 cases. American Journal of Surgical Pathology 200933 1886-1893. (doi:10.1097/PAS.0b013e3181bd535b)

30 Kishimoto M, Okimura Y, Kimura K, Mizuno I, Iguchi G, Fumoto M, Takahashi Y, Kanda F, Kaji H, Abe $\mathrm{H}$ et al. Multifocal fibrosclerosis as a possible cause of panhypopituitarism with central diabetes insipidus. Endocrine Journal 200047 335-342. (doi:10.1507/endocrj.47.335)

31 Braun J, Schuldes H, Berkefeld J, Zanella F, Jonas D, Usadel KH \& Badenhoop K. Panhypopituitarism associated with severe retroperitoneal fibrosis. Clinical Endocrinology 200154 273-276. (doi:10.1046/ j.1365-2265.2001.01023.x)

32 Fukuda W, Kimura M, Akaogi T, Sako M, Ohiwa K, Yamamoto Y, Kato G, Hayashi H \& Yoshikawa T. Multifocal fibrosclerosis: retroperitoneal fibrosis associated with a suprasellar tumor and pachymeningitis. Internal Medicine 200342 1006-1010. (doi:10.2169/ internalmedicine.42.1006)

33 Taniguchi T, Hamasaki A \& Okamoto M. A case of suspected lymphocytic hypophysitis and organizing pneumonia during maintenance therapy for autoimmune pancreatitis associated with autoimmune thrombocytopenia. Endocrine Journal 200653 563-566. (doi:10.1507/endocrj.K05-179)

34 Sommerfield AJ, Lockman KA, Bathgate AJ \& Patrick AW. Multifocal fibrosclerosis: a new case report. Annals of Clinical Biochemistry $2008 \mathbf{4 5}$ 99-101. (doi:10.1258/acb.2007.007049)

35 Nishimori I, Tamakoshi A \& Otsuki M \& Research Committee on Intractable Diseases of the Pancreas, Ministry of Health, Labour, and Welfare of Japan. Prevalence of autoimmune pancreatitis in Japan from a nationwide survey in 2002. Journal of Gastroenterology 200742 6-8. (doi:10.1007/s00535-007-2043-y)

36 Bates AS, Van't Hoff W, Jones PJ \& Clayton RN. The effect of hypopituitarism on life expectancy. Journal of Clinical Endocrinology and Metabolism 199681 1169-1172. (doi:10.1210/jc.81.3.1169)

37 Gao H, Gu YY \& Qiu MC. Autoimmune hypophysitis may eventually become empty sella. Neuro Endocrinology Letters 201334 102-106.

38 Lupi I, Zhang J, Gutenberg A, Landek-Salgado M, Tzou SC, Mori S \& Caturegli P. From pituitary expansion to empty sella: disease progression in a mouse model of autoimmune hypophysitis. Endocrinology 2011152 4190-4198. (doi:10.1210/en.2011-1004)

39 Shinji A, Sano K, Hamano H, Unno H, Fukushima M, Nakamura N, Akamatsu T, Kawa S \& Kiyosawa K. Autoimmune pancreatitis is closely associated with gastric ulcer presenting with abundant IgG4-bearing plasma cell infiltration. Gastrointestinal Endoscopy 200459 506-511. (doi:10.1016/S0016-5107(03)02874-8)

40 Deshpande V, Gupta R, Sainani N, Sahani DV, Virk R, Ferrone C, Khosroshahi A, Stone JH \& Lauwers GY. Subclassification of autoimmune pancreatitis: a histologic classification with clinical significance. American Journal of Surgical Pathology 201135 26-35. (doi:10.1097/PAS.0b013e3182027717)

41 Zen Y \& Nakanuma Y. IgG4-related disease: a cross-sectional study of 114 cases. American Journal of Surgical Pathology 201034 1812-1819. (doi:10.1097/PAS.0b013e3181f7266b)

Received 4 August 2013

Revised version received 21 October 2013

Accepted 28 October 2013 